\title{
ARTEFATOS HÍBRIDOS: expressões materiais do dinamismo cultural e questões de reflexão para o design
}

\author{
Marília Riul ${ }^{1}$ \\ Universidade de São Paulo - Instituto de Energia e Ambiente - Programa de Pós \\ Graduação em Ciência Ambiental \\ mriul@usp.br
}

Maria Cecília Loschiavo dos Santos

Universidade de São Paulo - Faculdade de Arquitetura e Urbanismo closchia@usp.br

Resumo: A nossa época se caracteriza pelos processos de interação cultural e entrecruzamento de realidades e seus efeitos nas várias dimensões da vida. A dimensão material da cultura toma parte dessas transformações, pelo menos em duas vias: os artefatos influenciam as culturas e estas, por sua vez, influenciam a concepção de artefatos. Este artigo trata do segundo caso, em que o dinamismo cultural exerce forças determinantes sobre a produção do mundo material. O foco do trabalho foi a análise da produção artesanal de artefatos utilitários em povoados da região da Barra do Rio Mamanguape, Paraíba, Brasil, sob o ponto de vista do seu dinamismo no decorrer da história. Os dados foram coletados em pesquisa de campo, através de entrevistas e registros fotográficos. O levantamento bibliográfico complementou a análise, que nos trouxe a compreensão de que mudanças socioculturais e em aspectos ambientais da região levaram a transformações significativas na produção artesanal de artefatos. Discutimos os significados dessas mudanças para o legado da cultura material local e iniciamos uma reflexão sobre as contribuições da compreensão desses processos para o design, como atividade configuradora do mundo material e influente nas complexas questões socioambientais.

Palavras-chave: Barra do Rio Mamanguape, produção artesanal, artefatos híbridos, dinamismo cultural, design

\begin{abstract}
Our time is peculiar for the cultural interaction and realities intercrossing processes and for their effects on the various dimensions of life. The material dimension of culture take part of these transformations, at least in two ways: artifacts influence cultures and cultures influence the
\end{abstract}

\footnotetext{
${ }^{1}$ Bolsista Fapesp - Processo Fapesp N²011/21336-1
} 
conception of artifacts. This paper addresses the second situation, which assumes that cultural dynamism affects significantly the material world production. The paper analyzes the craft production of utilitarian artifacts in traditional communities in the region of Barra do Rio Mamanguape, Paraiba, Brasil, from the viewpoint of cultural dynamism throughout history. Data was collected in field research, with semi-structured interviews and photographic registers. Bibliographic research complemented the analysis that brought us understanding that sociocultural and environmental changes in the studied region led to significant transformations in craft production. We discuss the meaning of these changes for the local material culture legacy and begin a reflection about what contributions the understanding of these processes can bring to design.

Keywords: Barra do Rio Mamanguape, craft production, hybrid artifacts, cultural dynamism, design

\section{INTRODUÇÃO}

Vários territórios distribuídos pelo Brasil são habitados por comunidades autóctones por um longo período de tempo - como é o caso dos pescadores artesanais e caiçaras do extenso litoral brasileiro, dos quilombolas e camponeses espalhados nas vastas áreas rurais do país e dos indígenas da Amazônia e de outros estados.

Essas populações caracterizam-se por um modo de vida distinto do padrão urbano-industrial e desenvolvem expressões culturais diferenciadas, com base nas peculiaridades das práticas sociais e do ambiente vivenciado (DIEGUES, 1994).

Assim, uma rica diversidade cultural ainda resiste fora do contexto das cidades brasileiras. Por outro lado, sobre essas variantes culturais avançam os processos de modernização e globalização, com seus diversos efeitos transformadores.

A cultura material é uma das dimensões da vida afetadas por essas mudanças e a produção artesanal de artefatos, característica das comunidades tradicionais, tem passado por grandes transformações (VAN VELTHEN, 1986; VIDAL \& SILVA, 1995).

Este artigo descreve as características atuais da produção artesanal de povoados existentes na região da Barra do Rio Mamanguape, na Paraíba, e a analisa do ponto de vista do dinamismo cultural. A pesquisa foi realizada por meio de levantamento de dados bibliográficos e documentais e de pesquisa de campo - através de entrevistas semi-estruturadas e registros fotográficos nas comunidades locais.

Mudanças socioculturais e em aspectos ambientais da região levaram a transformações significativas na produção artesanal de artefatos. Os produtos desse processo dinâmico são: por um lado, a continuidade pontual da produção de artefatos no estilo tradicional e o seu risco de desaparecimento; e por outro lado, a produção de artefatos híbridos, que representam a mestiçagem de práticas tradicionais com elementos inovadores advindos das novas práticas sociais ali desenvolvidas.

Discutimos os significados dessas mudanças para o legado da cultura material local e iniciamos uma reflexão sobre as contribuições da compreensão desses processos para o design, como atividade configuradora do mundo material e influente nas complexas questões socioambientais. 


\section{DESENVOLVIMENTO}

\subsection{Fundamentos teóricos}

\subsubsection{Design, ética e aspectos socioambientais}

Friedman (2002) define design como um processo que envolve a criação de algo novo (ou a reconfiguração de algo que já existe) para um propósito; de encontro a uma necessidade; para resolver um problema; ou para transformar uma situação menos desejável em uma preferível.

Para Fry (2009), o papel do design excede os objetivos de dar forma, funcionalidade e significado simbólico aos seus produtos. Fry $(2009$, p.30) escreve que "everything designed goes on designing". O autor atenta para a complexidade relacional do design que, além de conceitualmente e tecnicamente prefigurar a forma e as funções operacionais e simbólicas das coisas, concebe também o seu destino plural numa trajetória composta pelas escalas de espaço e tempo. Isso quer dizer que o design tem a tarefa de prever se os seus produtos terão uma vida útil funcional ou não, ou se serão agentes para o prejuízo ou benefício da humanidade, por exemplo.

Essa complexidade implícita na atividade do design agrega pressupostos éticos de profunda importância e vários autores a discutem sob a ótica da problemática socioambiental. As análises apontam para a importância de repensar essa atividade, já que ela tem se orientado mais para os objetivos de mercado e menos para as necessidades sociais e os problemas ambientais (PAPANEK, 1995; MARGOLIN, 2002; WALKER, 2002; MCCOY, 2003; FRY, 2009; HARLAND \& SANTOS, 2009; BONSIEPE, 2012).

Papanek (2009) alerta que o design tem se preocupado muito em satisfazer vontades e desejos efêmeros, enquanto necessidades humanas são rejeitadas. Tais criações ameaçam a segurança e a saúde da sociedade, seja através de artefatos inseguros no uso, ou pelo desperdício de recursos naturais insubstituíveis, pelo acúmulo de toneladas de resíduos decorrentes de mercadorias fugazes, ou pela escolha de materiais e processos produtivos poluidores (PAPANEK, 2009).

Assim, tais autores destacam a prevalência da racionalidade econômica, a neutralidade política e o abandono de valores éticos e das reais necessidades sociais que caracterizam a prática do design. Essa postura permeada pela racionalidade tecnocientífica, alimentada pela razão economicista, caracteriza o paradigma em que o design se insere para dar sua contribuição à produção do mundo material.

Por outro lado, há a expectativa de construção de um paradigma de saber articulado à complexidade socioambiental e atento à profunda crise que abala a humanidade. É princípio norteador dessa racionalidade a abertura à diversidade, à interdependência e à complexidade (GINZBURG, 1990; SANTOS, 2000; LEFF, 2002).

Nessa perspectiva, conhecer repertórios distintos de expressões culturais saberes, práticas, comportamentos e visões de mundo edificados a partir de diferentes contextos sociais e ambientais - mostra-se uma importante via para a diversificação da nossa forma de interpretar a realidade. A partir daí, são conservadas as possibilidades de conceber respostas consistentes e eficazes aos problemas atuais e futuros, inclusive no que diz respeito à concepção da nossa cultura material.

\subsubsection{Cultura: diversidade e dinamismo}


Viertler (2002) entende as culturas como "modos específicos ou padrões que regem a convivência e a sobrevivência social por um tempo mais ou menos prolongado". A autora considera que o comportamento humano não pode ser explicado isoladamente, mas sim em termos de ações e comportamentos sociais derivados de referenciais culturais específicos ao seu contexto social.

Vidal \& Silva (1995) explicam que a "cultura se compõe de idéias, concepções, significados, sempre reelaborados, ao longo do tempo e através do espaço e que seu dinamismo acompanha o da própria vida". Tais significados e concepções se expressam através das práticas sociais, do discurso, da fala, das manifestações artísticas de um povo ou através da criação dos artefatos incorporados à sua vivência.

A cultura humana se expressa numa multiplicidade de formas através do tempo e do espaço. Tal pluralidade reflete-se dos referenciais específicos próprios das particularidades do contexto social vivenciado e atrelam-se também à diversidade de ambientes em que se dá a ocupação humana (VIERTLER, 2002). Segundo a UNESCO (2002, p.2),

\footnotetext{
Essa diversidade se manifesta na originalidade e na pluralidade de identidades que caracterizam os grupos e as sociedades que compõem a humanidade. Fonte de intercâmbios, de inovação e de criatividade, a diversidade cultural é, para o gênero humano, tão necessária como a diversidade biológica para a natureza.
}

Para Durham (2004), os fenômenos culturais devem ser analisados sob a perspectiva da dinâmica cultural, "do processo permanente de reorganização das representações na prática social, representações estas que são simultaneamente condição e produto desta prática" (DURHAM, 2004, p. 231). A autora salienta que os portadores da cultura a reconstituem através de sua elaboração da realidade.

Ao dinamismo intrínseco às culturas, somam-se os processos de globalização cultural e modernização, como fenômenos de elevada influência na configuração e reconfiguração dos códigos simbólicos e práticas sociais.

$\mathrm{Na}$ modernização, segundo Little (2002), está implícito o processo de industrialização das economias, através de novas tecnologias produtivas e da transformação de formas "tradicionais" de organização social em "sociedades modernas" e, no caso do capitalismo, fundadas nos princípios do liberalismo. Assim, a modernização, entendida como a transição das sociedades tradicionais para as sociedades modernas, está vinculada à idéia de desenvolvimento econômico e fundada no paradigma ocidental de progresso.

Já o processo de globalização atua na integração e conexão de comunidades e organizações, a partir da compressão de distâncias e escalas temporais. Essa integração global se dá pela comunicação e pelo consumismo global, caracterizando um "supermercado cultural", em que identidades se tornam desvinculadas de tempos, lugares, histórias e tradições específicas (HALL, 2005).

\subsubsection{Comunidade tradicionais}

Grande parte das florestas tropicais e outros ecossistemas ainda não degradados pela atividade humana são habitados por grupos sociais com características culturais e modos de vida peculiares, distintos da maneira de viver das sociedades urbanas e industrializadas (DIEGUES, p. 71, 1994). 
Essas sociedades são comumente designadas de sociedades tradicionais, comunidades tradicionais ou populações tradicionais. Arruda (1999) emprega o termo "sociedades tradicionais" referindo-se a grupos humanos de cultura distinta, que reproduzem seu modo de vida historicamente e que vivem de forma mais ou menos isolada, baseada na cooperação social.

Esses grupos diferenciam-se das sociedades urbanas, segundo Diegues (1994), por estarem associados a um modo de produção em que a força de trabalho e a própria natureza não são vistos como objeto de compra e venda (mercadorias) e onde há uma grande dependência dos recursos naturais e dos ciclos da natureza. Tais sociedades desenvolvem-se no contexto da pequena produção mercantil, onde a dependência do mercado já existe, mas não é total, e suas atividades não visam diretamente ao lucro (DIEGUES, 1994).

São características das comunidades tradicionais: a) dependência da natureza, dos ciclos naturais e dos recursos naturais com os quais constroem um modo de vida; b) conhecimento aprofundado da natureza e de seus ciclos, transmitido através da oralidade, que se reflete em estratégias de uso e manejo dos recursos naturais; c) noção do território ou espaço onde o grupo social se reproduz; d) moradia e ocupação desse território por várias gerações; e) importância das atividades de subsistência; f) reduzida acumulação de capital; g) importância dada à unidade familiar e às relações de parentesco ou compadrio; h) importância das simbologias, mitos e rituais; i) tecnologia simples, de impacto limitado sobre o meio ambiente (DIEGUES, 1994).

$O$ conhecimento ecológico tradicional (no inglês traditional ecological knowledge, TEK) fornece as bases para as práticas de uso de recursos em sociedades não industriais. Define-se como "um corpo cumulativo de conhecimentos e crenças, transmitidos através das gerações pela transmissão cultural, sobre as relações entre os seres vivos (inclusive humanos) entre si e com o ambiente" (BERKES, 1993).

O TEK pode ser dividido em quatro níveis (BERKES, 1999 apud NORDI, 2000): a) conhecimento de plantas e animais (identificação, distribuição e comportamento); b) sistema de manejo dos recursos (conjunto de práticas, ferramentas e técnicas); c) conjunto de regras de interação social (preocupação coletiva); e d) visão do mundo em que impera uma ordem conceitual que inclui religião, ética, sistemas de crença etc.

Berkes (1993) aponta uma série de diferenças entre o conhecimento ecológico tradicional e o conhecimento científico ocidental. Dentre elas, a natureza holística do conhecimento tradicional e a sua propriedade inclusiva com relação ao sistema ético e moral, em parte baseado na noção de não separação entre "natureza" e "cultura", que se configura distante do objetivo de controle da natureza pelo homem.

Nordi (2000) afirma que a ciência ocidental separa, por princípio, fato e valor. Elimina do seu meio toda a competência ética e baseia sua objetividade na eliminação do sujeito do conhecimento científico. Enquanto que o conhecimento tradicional possui componentes intuitivos, espirituais e morais (BERKES, 1993).

\subsubsection{Produção artesanal e design vernacular}

Produção artesanal, ou artesanato, segundo Martins (1978), se refere ao regime de trabalho caracterizado pelas técnicas do artesão e pelo amplo uso das mãos, por vezes com o auxílio de ferramentas simples. Segundo o autor, "artesão é a pessoa que faz, a mão, objetos de uso frequente na comunidade. Seu aparecimento foi resultado da pressão da necessidade sobre a inteligência, aliada ao poder de inovar". 
Como explica Morales (2008) no caso do artesão, a função projetual e a função produtiva são articuladas pela mesma pessoa, ao passo em que, no design industrial, essas duas funções foram separadas.

O artesão é o sujeito de todo o processo de produção, articulado em várias etapas de execução. Assim, o ofício se particulariza pela concentração do trabalho em uma só pessoa (contrária à divisão do trabalho) e ainda pela não separação entre elaboração mental e trabalho manual. Nessa atividade, o produtor tem o conhecimento e domínio de todo o processo, inteiramente realizado pelo seu gesto e guiado por sua "elaboração consciente" (MARTINS, 1978).

Os produtos do artesanato são peças originais que expressam a subjetividade de seu produtor e as particularidades culturais em que se contextualizam, além de refletirem também caracteres econômicos, sociais, políticos, ambientais e tecnológicos (MORALES, 2008).

A produção artesanal, segundo Morales (2008), ocorre em dois contextos diferentes: o dos camponeses e indígenas, em que os objetos são produzidos em complementaridade às atividades agrícolas e ao trabalho doméstico, com baixo investimento em matéria-prima e para atendimento das necessidades locais; e a produção voltada para mercados especializados, que atende a maiores demandas.

As culturas tradicionais e os recursos naturais locais geralmente oferecem uma identidade particular à produção artesanal realizada nos povoados. A criação desses objetos integra tanto o conhecimento histórico local, ou de sua etnia, como o conhecimento técnico sobre o uso dos recursos do entorno e a expressão individual de quem produz (MORALES, 2008).

No campo do design utiliza-se o termo design vernacular para tratar da produção artesanal de artefatos. Assim como, as expressões "design não-profissional" (PACEY, 1992), "pré-design" (MAGALHÃES, 1997) e "design espontâneo" (SANTOS, 2003), o termos refere-se às soluções materiais independentes do contexto acadêmico ou de qualificações institucionais (LAWSON, 1980 apud REITAN, 2007).

Na visão de Walker (2002), design vernacular refere-se à produção de artefatos pelas culturas tradicionais, caracterizada pela criatividade, uso de recursos limitados e disponíveis em seu ambiente e com forte valor simbólico freqüentemente embutido nos objetos, cujos valores transcendem os benefícios funcionais.

Já Santos (2003) desdobrou esse tema com foco em grupos sociais urbanos e entendeu "design espontâneo" como a "prática de resistência criativa de procurar soluções engenhosas aplicáveis à resolução de problemas concretos, num contexto de severa falta de recursos" (SANTOS, 2003).

\subsection{Métodos}

As técnicas de coletas de dados foram os levantamentos bibliográfico e documental e a pesquisa de campo. Em campo foram utilizadas os procedimentos da entrevista, da observação direta e dos registros fotográficos.

Com as entrevistas foi possível caracterizar a produção artesanal da região, assim como obter outras informações pertinentes de forma espontânea no decorrer de cada relato oral. Além de roteiros semi-estruturados, foi utilizado um banco de imagens, isto é, um conjunto de fotografias de artefatos artesanais característicos da região Nordeste e do contexto litorâneo. Esse instrumento foi utilizado para facilitar a interação com o entrevistado e a sua compreensão em relação ao objeto de estudo. 
A amostragem foi intencional, adequada à busca específica por artesãos e artefatos. A investigação foi realizada com a ajuda de um informante-chave nos seguintes povoados: Barra de Mamanguape, Lagoa de Praia, Praia de Campina, Tanques, Sítio Saco, Tavares, Cravassu, Rio Tinto, Camurupim e Tramataia.

\subsection{1 Área de estudo}

A área de estudo está situada na Bacia Hidrográfica do Rio Mamanguape, no litoral norte, e abrange porções do território dos municípios de Rio Tinto e Lucena, no estado da Paraíba, Brasil. Observa-se uma rica diversidade de ambientes nessa região: remanescentes de mata atlântica e restinga, arrecifes costeiros, dunas, falésias, ilhas, croas, camboas e apicuns (NISHIDA, 2000).

Os povoados dali caracterizam-se pela mistura cultural dos indígenas potiguaras, negros e europeus. Formaram-se modos de vida adaptados às peculiaridades dos espaços ocupados e às relações estabelecidas com os recursos naturais disponíveis. A maioria das comunidades está situada em ambiente rural e interage diretamente com os ecossistemas. Praticam a agricultura de subsistência, a coleta de frutos, a extração de madeira, a pesca e o extrativismo de moluscos e crustáceos (CUNHA et al., 1992; EMBRAPA, 2008).

No ano de 1993, foi criada a Área de Proteção Ambiental (APA) da Barra do Rio Mamanguape, uma unidade de conservação que abrange 16.400 ha dos territórios dos municípios de Rio Tinto e Lucena. O principal objetivo da APA é a conservação do habitat do peixe-boi marinho e das áreas de manguezal e mata atlântica que ali permaneceram com o passar da história (BRASIL, 1993).

\subsection{Resultados e discussão}

A produção artesanal atual dos vilarejos situados na região da Barra do Rio Mamanguape, assim como no passado, articula-se profundamente com as atividades domésticas e de subsistência ali praticadas. A vida e o ambiente doméstico frequentemente são penetrados pelas atividades de trabalho, o que faz comum o compartilhamento de artefatos utilitários nessas duas esferas.

Como explicou Martins (1978), a necessidade de atender a uma função e a frequência de sua incidência determinam a produção de um artesão em uma comunidade. Nesse caso, as atividades de subsistência, a pesca e a agricultura, fizeram surgir uma cultura material integrada ao trabalho cotidiano nos ambientes de pesca e da roça e também para o consumo dos produtos dessas atividades produtivas no ambiente doméstico.

A produção de artefatos também está diretamente relacionada aos recursos naturais existentes na região (MARTINS, 1978; VIDAL \& SILVA, 1995). Na região estudada, os principais materiais vêm das matas, e são recursos vegetais tradicionalmente utilizados através das gerações, por meio de técnicas e conhecimentos detalhados sobre as plantas e ambientes específicos.

Essa vocação natural resultou numa produção artesanal caracterizada pela fisionomia dos trançados, técnica que tem origem na interação íntima das diversas tribos indígenas brasileiras e a natureza e que remete ao início da história da humanidade (VIDAL \& SILVA, 1995).

Os artefatos são produzidos com várias técnicas de entrançamento das fibras vegetais e o seu uso no cotidiano também é diversificado. Nas culturas indígenas, a 
funcionalidade dos objetos produzidos com essa técnica variam entre o armazenamento de pertences individuais, o transporte e o processamento de alimentos e também o adorno pessoal (VAN VELTHEN, 2007).

Outros usos foram atribuídos aos artefatos locais (ver Figura 1), como armazenar alimentos na cozinha e guardar uma variada gama de itens domésticos ou de trabalho, e capturar peixes e caranguejos. Tais objetos são destinados ao autoconsumo pelos artesãos ou produzidos sob encomenda para vizinhos e familiares.

As expressões materiais da cultura local são também reflexo da mistura cultural desdobrada na região, com fortes influências dos seus primeiros habitantes, os indígenas potiguaras, além dos elementos culturais europeus e africanos que se juntaram posteriormente.

Outros processos culturais que ali se desenrolaram foram determinantes para as feições atuais dessa atividade artesanal. As transformações realizadas pelo homem no ambiente e na sua configuração territorial no decorrer da história, assim como os reflexos socioculturais e econômicos dos processos de modernização e globalização que ali atuam, agiram como importantes modeladores das práticas de produção de artefatos artesanais.

As mudanças ambientais e na configuração do território desencadearam principalmente efeitos no acesso aos recursos naturais e ambientais, tanto no que diz respeito aos recursos vegetais essenciais à produção artesanal, quanto àqueles necessários à reprodução do modo de vida tradicional local, permeado pelo uso de artefatos produzidos localmente.

As transformações no ambiente se devem em grande medida à devastação de enormes áreas de Mata Atlântica a partir dos anos 1970 para a implantação da monocultura da cana de açúcar. A expansão da atividade canavieira promoveu o desmatamento de vastas áreas de matas, limitando o acesso dos artesãos à matériaprima, tanto pela considerável diminuição das áreas de mata, quanto pelas longas distâncias dos fragmentos remanescentes.

A esses obstáculos, somam-se ainda as medidas gestoras de preservação ambiental recentemente determinadas para essa região, motivadas pelas ameaças advindas da exploração excessiva dos canavieiros no território historicamente habitado por pequenos povoados que ali reproduziam a sua cultura de subsistência.

As limitações dos habitantes locais ocorrem também com relação às suas atividades tradicionais de subsistência. O avanço dos canaviais, a especulação imobiliária e as medidas de conservação do ambiente criadas para a região reduziram drasticamente as práticas de agricultura de subsistência.

$\mathrm{O}$ acesso restrito e a reduzida disponibilidade de recursos naturais (matériasprimas e terra), junto ao desaparecimento de atividades produtivas características da região, alavancaram mudanças profundas no modo de vida local, como a transferência dos habitantes locais para o trabalho assalariado. Tais transformações refletiram-se no quase desaparecimento do ofício de artesão, diminuindo notavelmente a produção dos artefatos utilizados tradicionalmente no cotidiano local.

Um exemplo dessa realidade é a queda drástica da produção da mandioca e dos alimentos processados a partir desse vegetal. Isso levou ao desuso e desaparecimento das casas de farinha, assim como fez rarear a produção das urupemas (ver Figura 1, item [4]), as peneiras circulares utilizadas principalmente 
nesse processo produtivo e na produção de alimentos derivados da mandioca (farinha, tapioca, beiju) e do milho.

Dentre os artefatos tradicionais que foram encontrados temos também as cestas e os cestos ou balaios (ver Figura 1, itens [3] e [6]), antigamente muito utilizados na agricultura para transportar a produção. Essa permanência se deve talvez à grande versatilidade funcional desses objetos, que varia entre o armazenamento de pertences pessoais, transporte e tratamento do pescado, armazenamento de alimentos no ambiente doméstico, decoração e outros usos diversos, apresentando-se em diversas dimensões e formas.

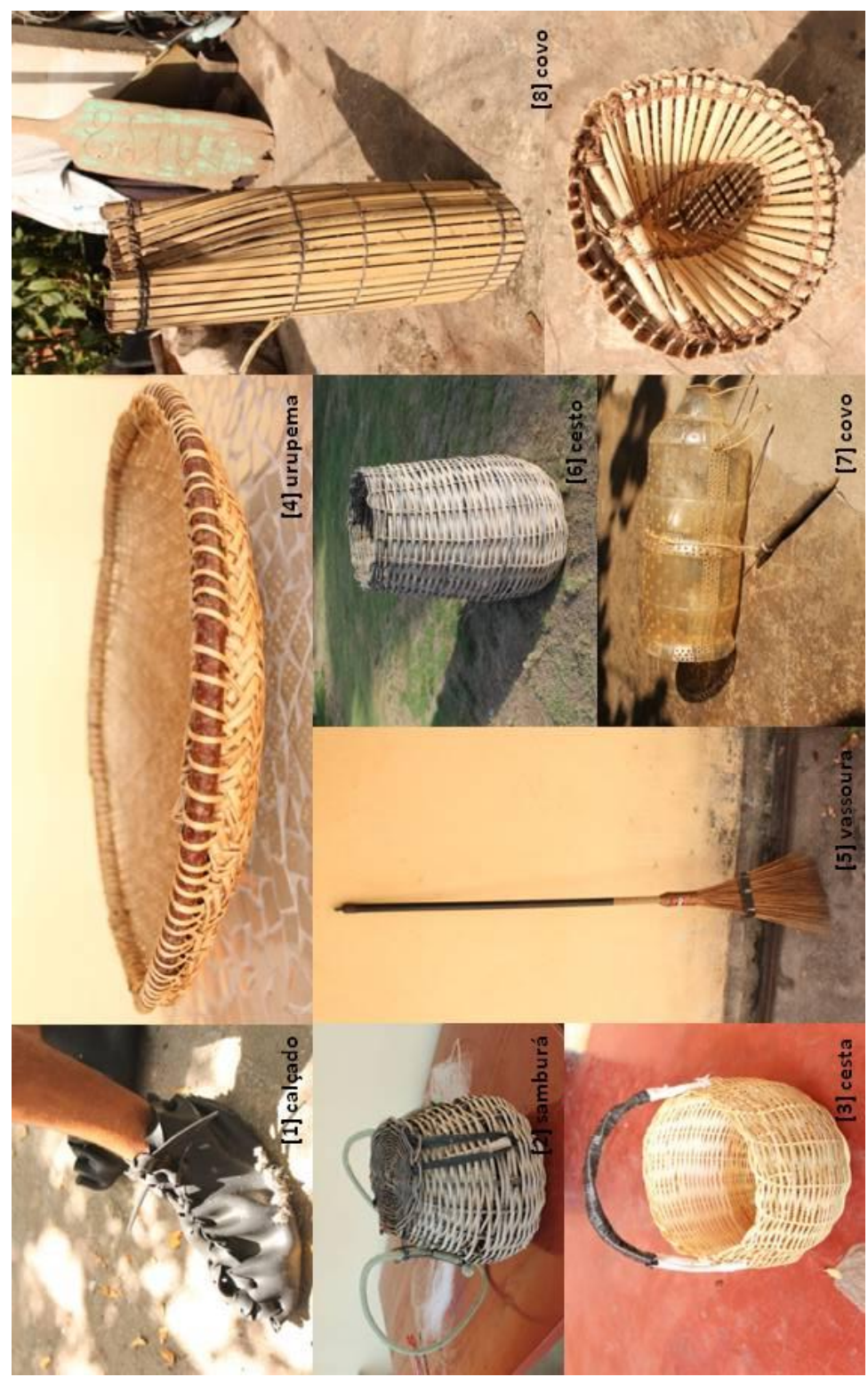

Figura 1: Artefatos da produção artesanal na região da Barra do Rio Mamanguape Fonte: Elaborada pela autora, com base na pesquisa realizada

O samburá (ver Figura 1, item [2]) é uma variação dos cestos que possui tampa e alça para ser utilizado a tiracolo pelo pescador. Enquanto o trabalho continua, o pescado armazenado no recipiente permanece vivo por estar mergulhado na água, mantendo-se fresco para o consumo. Segundo os artesãos, a sua produção é mais difícil devido à variação do diâmetro do seu corpo. Esse artefato é mais raro do que os 
cestos sem tampa, devido a sua função específica na pesca, em que tem sido substituído pelo uso de sacolas, baldes e outros recipientes plásticos.

Apesar das mudanças notadas, como o uso predominante das redes de pesca industrializadas, armadilhas de pesca também são produzidas artesanalmente, como é o caso do covo (ver Figura 1, item [8]).

O covo é um artefato tradicional utilizado para a captura do amoré, espécie de peixe de mangue, e do caranguejo. Esse artefato tem formato cilíndrico, com uma das extremidades achatada e onde também se encaixa uma tampa (por onde se retira o pescado ao final do seu uso). A outra extremidade é circular e possui a "sangra", um elemento cônico que permite a entrada das presas, mas que dificulta a sua saída, pois possui pontas afiadas. Esse processo é complementado pelo posicionamento do objeto a favor da correnteza do rio.

No tempo passado, o covo era feito apenas com o uso de fibras vegetais. Hoje percebemos a combinação dessas fibras com o reaproveitamento de materiais industrializados, como os cordões de náilon encontrados na praia e usados na amarração.

Uma nova versão do covo foi identificada (ver Figura 1, item [7]) em campo. Esse artefato foi produzido com a reutilização de garrafas PET, perfurado em todo o seu comprimento, para permitir a sua funcionalidade.

Já o calçado (ver Figura 1, item [1]) representa a combinação da antiga necessidade de proteger os pés, relacionada à catação de caranguejo no agressivo ambiente do manguezal, com a inovação material da câmara de ar de pneus.

A nova versão do covo, a vassoura (ver Figura 1, item [5]), bem como outros artefatos observados em campo, combinam conhecimentos e técnicas tradicionais, recursos naturais locais, novos materiais disponíveis (resíduos e outros elementos industrializados) e as inovações de saberes e técnicas destes decorrentes. Tais "artefatos híbridos" (BURKE, 2003) representam uma adaptação às transformações ambientais e socioculturais na dimensão material da cultura da região.

As principais implicações da dinâmica sociocultural e ambiental analisada para a cultura material da região incluem, por um lado, o rareamento do ofício de artesão e até o desaparecimento de itens da cultura material tradicional e, por outro lado, o surgimento da produção hibridizada de utensílios que, contextualizados no dinamismo cultural, permanecem necessários no cotidiano dos seus produtores.

O legado da produção artesanal estudada representa uma tentativa insistente de reprodução social e cultural autônoma, diante da influência de tantos fatores que a inibem e que a tornam dependente do contexto urbano, industrial e globalizado. Os artefatos encontrados refletem a mestiçagem rural/urbana, local/globalizada, natural/industrializada e materializam soluções para situações cotidianas de subsistência e da vida doméstica.

\section{CONSIDERAÇÕES}

A análise realizada a respeito da dinâmica da produção artesanal de artefatos na região da Barra do Rio Mamanguape permitiu relacionar processos socioambientais e culturais complexos e a produção do mundo material.

Pudemos ilustrar que a produção de artefatos se adapta a processos críticos, como a escassez de recursos e a privação de um modo de vida tradicional de uma região, e persiste no seu objetivo básico de facilitar as atividades humanas. 
Vivenciamos notáveis processos críticos socioambientais e a esses processos a complexidade é inerente. Se o design é a atividade projetual especializada responsável por grande parcela da produção de objetos no mundo urbano e industrial, temos o alerta de que é necessário investir profundamente na formação de designers capazes de formular propostas à altura da complexidade intensificada pela nossa condição.

Parece um desafio atual para o design racionalizar a complexidade do mundo e superar os problemas de compreensão e análise advindos da separação entre função projetual e função produtiva no exercício da produção do mundo material. Assim, parece pertinente inserir o design no atual desafio epistêmico e criativo de formular conhecimentos que tentem melhor compreender a realidade, com os objetivos de diminuir o empobrecimento ocasionado pela exagerada especialização do saber e aumentar a frequência de soluções consistentes e preocupadas com as questões relevantes da nossa época.

\section{REFERÊNCIAS}

ARRUDA, R. Populações Tradicionais e a proteção dos recursos naturais em Unidades de Conservação. Ambiente \& Sociedade, Ano II, N. 5, 1999.

BERKES, F. Traditional Ecological Knowledge in Perspective. In: Julian T. Inglis (ed.). Traditional Ecological Knowledge - Concepts and Cases. International Program on Traditional Ecological Knowledge Canadian Museum of Nature. 1993.

BONSIEPE, G. Design e crise. Agitprop: Revista Brasileira de Design. Ano IV, n. 44. 2012. BRASIL. Decreto Federal no 924, de 10 de setembro de 1993.

BURKE, P. Hibridismo cultural. Editora Unisinos, Rio Grande do Sul. 2003.

CUNHA ET AL. Reserva extrativista para regiões de mangue: uma proposta preliminar para o Estuário de Mamanguape - (Paraíba). Programa de Pesquisa e Conservação de Áreas Úmidas no Brasil - Pró-reitoria/USP. International Development Research Centre (IDRC)/Fundação Ford. São Paulo, 1992.

DIEGUES, A. C. O Mito Moderno da Natureza Intocada. NUPAUB/USP/Hucitec. São Paulo. 1994.

DURHAM, E. R. A dinâmica cultural na sociedade moderna. In: DURHAM. Eunice Ribeiro. A dinâmica da cultura: ensaios de antropologia. Org. Omar Ribeiro Thomaz. São Palo: Cosac Naify. 2004.

EMBRAPA MEIO AMBIENTE, Avaliação de impactos ambientais para gestão da APA da Barra do Rio Mamanguape/PB. Jaguariúna: Embrapa Meio Ambiente, 2008.

FRIEDMAN, K. Theory construction in design research: criteria, approaches, and methods. In: SHACKLETON, J. \& DURLING, D. (eds.) Common ground: Proceedings of the 2002 Design Research Society International Conference. Londres, Reino Unido, 5-7 de setembro, p.388-414. 2002.

FRY, Tony. Design futuring: sustainability, ethics, and new practice. Oxford: Berg, 2009. GINZBURG, C. Mitos, emblemas, sinais: Morfologia e História. São Paulo: Companhia das Letras. 1990.

HALL, S. A identidade cultural na pós-modernidade. Rio de Janeiro, DP\&A, 2005. HARLAND, R. \& SANTOS, M.C.L. From greed to need: notes on human-centred design. IN: Interrogations: Creative Interdisciplinarity in Art and Design Research: Proceedings of AHRC Postgraduate Conference 2009, pp. 141-158

LEFF, E. Epistemologia ambiental. São Paulo: Cortez. 2002 
LITTLE, Paul. E. Etnodesenvolvimento Local: autonomia cultural na era do desenvolvimento global. Tellus, ano 2, n.3, p.33-51. Campo Grande, 2002.

MAGALHAES, A. E Triunfo?: A questão dos bens culturais no Brasil. Rio de Janeiro: Nova Fronteira-Fundação Roberto Marinho, 1997.

MARGOLIN, V. The politics of the artificial: essays on design and design studies. Chicago: The University of Chicago Press, 2002.

MARTINS, S. Contribuição ao estudo científico do artesanato. Imprensa Oficial de Minas Gerais. Belo Horizonte, 1973.

McCOY, K. Good citizenship: design as a social and political force. In: HELLER, S; VIENNE, V. (eds). Citizen Designer: perspectives on design responsibility. Nova lorque: Allworth Press, 2003.

MORALES, F. S. Diseño e artesanía. In: FERNÁNDEZ, S. \& BONSIEPE, G. Historia del diseño en América Latina y el Caribe: Industrialización y comunicación visual para la autonomia. Editora Blücher. São Paulo, 2008.

NISHIDA, A. K. "Catadores de moluscos do litoral Paraibano: estratégias de subsistência e formas e percepção da natureza". Programa de Pós-Graduação em Ecologia e Recursos Naturais. Universidade Federal de São Carlos, São Carlos, SP, 2000. NORDI, N. Relação entre conhecimento tradicional e o conhecimento científico. Mesa redonda. III Simpósio de Etnobiologia e Etnoecologia, Piracicaba, jul. 2000.

PACEY, Philip. 'Anyone designing anything?' Non-professional designers and the history of design. In. Journal of Design History, vol. 5, n.3, 1992, p. 217 - 225.

PAPANEK, V. Arquitectura e design: ecologia e ética. Lisboa: Edições 70, 1995.

PAPANEK, V. Design for the Real World: Human ecology and social change. Chicago: Academy Chicago Publishers, 2009.

REITAN, J. B. Improvisation in Tradition: A Study of Contemporary Vernacular Clothing Design Practiced by Iñupiaq Women of Kaktovik, North Alaska. Tese de doutorado. Oslo School of Architecture and Design. Oslo, 2007.

SANTOS, B. S. A crítica da razão indolente: contra o desperdício da experiência. São Paulo: Cortez, 2000.

SANTOS, M. C. L. As cidades de plástico e papelão. Tese de Livre-docência. São Paulo, FAU-USP, 2003.

UNESCO. United Nations Educational, Scientific and Cultural Organization. Declaração Universal sobre a Diversidade Cultural. UNESCO, 2002.

VAN VELTHEM, L. H. Equipamento doméstico e de trabalho. In: RIBEIRO, D. Suma Etnológica Brasileira. V. 2, Tecnologia Indígena. Petrópolis: Vozes, 1986.

VAN VELTHEN, L. H. Trançados indígenas norte amazônicos: fazer, adornar, usar. Revista de Estudos e Pesquisas, FUNAI, Brasília, v.4, n.2, p.117-146, dez. 2007.

VIDAL, L. B. \& SILVA, A. L. O Sistema dos Objetos nas Sociedades Indígenas: Arte e Cultura. In: SILVA, A. L. e GRUPIONI, L. D. B. (ORG.) A Temática Indígena na Escola. Brasília, MEC/MARI/UNESCO. 1995.

VIERTLER, R. B. Métodos antropológicos como ferramenta para estudos em etnobiologia e etnoecologia. In: AMOROZO, M. C. M.; MING, L. C.; SILVA, S. P. (Org.) Métodos de coleta e análises de dados em etnobiologia, etnoecologia e disciplinas correlatas. CNPq, UNESP, 2002.

WALKER, S. A journey in design: an exploration of perspectives for sustainability. The Journal of Sustainable Product Design. N. 2, p. 3-10, Netherlands: Kluwer Academic Publishers, Netherlands, 2002. 